\title{
Numerical evidence for universality in the relaxation dynamics of near-extremal Kerr-Newman black holes
}

\author{
Shahar Hod ${ }^{1,2, a}$ \\ ${ }^{1}$ The Ruppin Academic Center, Emeq Hefer 40250, Israel \\ 2 The Hadassah Institute, Jerusalem 91010, Israel
}

Received: 20 November 2015 / Accepted: 10 December 2015 / Published online: 22 December 2015

(C) The Author(s) 2015. This article is published with open access at Springerlink.com

\begin{abstract}
The coupled gravitational-electromagnetic quasinormal resonances of charged rotating Kerr-Newman black holes are explored. In particular, using the recently published numerical data of Dias et al. (Phys Rev Lett 114:151101, 2015), we show that the characteristic relaxation times $\tau \equiv$ $1 / \Im \omega_{0}$ of near-extremal Kerr-Newman black holes in the regime $Q / r_{+} \leq 0.9$ are described, to a very good degree of accuracy, by the simple universal relation $\tau \times T_{\mathrm{BH}}=\pi^{-1}$ (here $Q, r_{+}$, and $T_{\mathrm{BH}}$ are respectively the electric charge, horizon radius, and temperature of the Kerr-Newman black hole, and $\omega_{0}$ is the fundamental quasinormal resonance of the perturbed black-hole spacetime).
\end{abstract}

\section{Introduction}

The influential uniqueness theorems [1-7] have revealed that all asymptotically flat stationary black-hole solutions of the coupled Einstein-Maxwell theory are uniquely described by the Kerr-Newman spacetime metric [8-10]. These elegant theorems therefore suggest that an asymptotically flat perturbed black-hole spacetime would eventually relax into a stationary Kerr-Newman solution, which is characterized by only three externally observable conserved parameters: the black-hole mass $M$, its angular momentum $J \equiv M a$, and its electric charge $Q$.

The response of a perturbed black-hole spacetime to external perturbations is characterized by a unique set of damped (complex) oscillations, known as black-hole 'quasinormal ringing'. These damped spacetime resonances are the characteristic 'sound' of the black-hole spacetime itself. They have therefore attracted considerable interest over the last four decades from both physicists and mathematicians (see [11-13] for excellent reviews).

a e-mail: shaharhod@gmail.com
The characteristic black-hole quasinormal resonances correspond to linearized wave fields propagating in the curved spacetime with the boundary conditions of purely outgoing waves at spatial infinity and purely ingoing waves crossing the black-hole horizon [14]. These physically motivated boundary conditions single out a discrete spectrum (here $m$ and $l$ are respectively the azimuthal harmonic index and the spheroidal harmonic index of the black-hole perturbation mode) $\{\omega(n ; m, l)\}_{n=0}^{n=\infty}$ of complex (damped) blackhole resonances. The fundamental (least damped) blackhole quasinormal resonance determines the characteristic timescale

$\tau_{\text {relax }} \equiv 1 / \Im \omega(n=0)$

for the decay (relaxation) of generic perturbation modes in the black-hole spacetime.

It should be emphasized that in most situations of physical interest, the black-hole eigen-frequencies (the characteristic black-hole quasinormal resonances) are not known in a closed analytical form. In particular, for most black-hole spacetimes one is forced to solve the black-hole perturbation equations numerically in order to explore the physical properties of the complex resonance spectra.

Near-extremal Kerr black holes are unique in this respect. In particular, their fundamental resonances are characterized by the remarkably simple analytical relation [15-29]:

$\Im \omega(n)=2 \pi T_{\mathrm{BH}}\left(n+\frac{1}{2}-i \delta\right) ; n=0,1,2, \ldots$,

where (we use natural units in which $G=c=\hbar=1$; here $M, M a, Q$, and $r_{ \pm}$are respectively the mass, angular momentum, electric charge, and horizon-radii of the black hole)

$$
T_{\mathrm{BH}}=\frac{r_{+}-r_{-}}{4 \pi\left(r_{+}^{2}+a^{2}\right)}
$$


is the Bekenstein-Hawking temperature of the black hole, and $\delta$ is the characteristic eigenvalue of the angular blackhole perturbation equation [the angular Teukolsky equation. See [30] for details. See, in particular, equations (2.7) and (6.3) of [30]].

The remarkably compact formula (2) was derived analytically in $[15,16]$. It is worth noting that this formula is univer$\mathrm{sal}$ in the sense that it is independent of the spin-parameter $s$ of the perturbation mode. In particular, the relation (2) provides a quantitative universal description for the relaxation properties of gravitational $(s=2)$, electromagnetic $(s=1)$, and scalar $(s=0)$ perturbation fields in the rotating neutral Kerr black-hole spacetime.

As for the more general case of charged rotating KerrNewman black holes, the simple relation (2) was established analytically only for the simplest case of scalar $(s=0)$ perturbation fields. Much less is known about the quasinormal resonance spectra associated with the coupled gravitational-electromagnetic perturbations of generic (rotating and charged) Kerr-Newman black holes. Our limited knowledge about the quasinormal spectra of generic KerrNewman black holes is a direct consequence of the fact that all attempts to decouple the gravitational and electromagnetic perturbations of charged rotating Kerr-Newman black-hole spacetimes have so far failed [8].

Recently, there have been some important numerical studies of the quasinormal resonance spectra which characterize the charged rotating Kerr-Newman black-hole spacetimes [31-35] (using the numerical results of [34], it was shown in [35] that charged Kerr-Newman black holes, like neutral Kerr black holes [15], are characterized by the simple relation $\Re \omega \rightarrow m \Omega_{\mathrm{H}}$, where $\Omega_{\mathrm{H}}$ is the angularvelocity of the black-hole horizon). In particular, in a very interesting work, Dias, Godazgar, and Santos [36] have recently provided detailed numerical results for the characteristic quasinormal resonances associated with the coupled gravitational-electromagnetic perturbations of generic KerrNewman black holes.

In the present paper we shall analyze these numerically computed [36] complex black-hole resonances. In particular, we shall show that the coupled gravitational-electromagnetic perturbations of near-extremal Kerr-Newman black holes are described extremely well by the relation (2) (it is worth emphasizing again that, for charged rotating Kerr-Newman black holes, the simple relaxation spectrum (2) was derived analytically only for the particular case of scalar $(s=0)$ perturbation modes. This restriction to the scalar case is a direct consequence of the fact that all attempts to decouple the gravitational and electromagnetic perturbations of generic (that is, charged and rotating) Kerr-Newman black holes have so far failed [8]). This interesting result (to be established below) suggests that neutral Kerr black holes and charged
Table 1 Quasinormal resonances of near-extremal charged rotating Kerr-Newman black holes. The data shown refer to the fundamental $l=m=2$ gravitational-electromagnetic perturbation mode with $\Im \omega_{0}=0.01 \mathrm{M}^{-1}[36]$. We display the dimensionless ratio $\Im \omega_{0} / \pi T_{\mathrm{BH}}$ for various values of the dimensionless black-hole charge parameter $Q / r_{+}$, where $T_{\mathrm{BH}}=\left(r_{+}-r_{-}\right) / 4 \pi\left(r_{+}^{2}+a^{2}\right)$ is the temperature of the corresponding Kerr-Newman black hole. One finds that, for nearextremal Kerr-Newman black holes in the regime $Q / r_{+} \leq 0.9$ (this is the regime of black-hole electric charges studied numerically in [36]), the quasinormal resonance spectra are characterized, to a good degree of accuracy, by the universal relation $\Im \omega_{0} / \pi T_{\mathrm{BH}} \rightarrow 1^{-}$

\begin{tabular}{ll}
\hline$Q / r_{+}$ & $\Im \omega_{0} / \pi T_{\mathrm{BH}}$ \\
\hline 0.2 & 0.978 \\
0.3 & 0.987 \\
0.4 & 0.972 \\
0.5 & 0.980 \\
0.6 & 0.945 \\
0.7 & 0.987 \\
0.8 & 0.972 \\
0.9 & 0.999 \\
\hline
\end{tabular}

Kerr-Newman black holes share the same universal relaxation properties in the near-extremal $T_{\mathrm{BH}} \rightarrow 0$ regime.

\section{Coupled gravitational-electromagnetic resonances of Kerr-Newman black holes}

Most recently, Ref. [36] has provided detailed numerical results for the fundamental (least damped) quasinormal resonances of charged rotating Kerr-Newman black holes. We have examined these numerically computed black-hole resonances in an attempt to reveal a possible universal pattern which characterizes the relaxation properties of these charged rotating black-hole spacetimes.

In Table 1 we present the black-hole resonances of nearextremal Kerr-Newman black holes [36]. The data shown in Table 1 refer to the fundamental $l=m=2$ (here $m$ and $l$ are respectively the azimuthal harmonic index and the spheroidal harmonic index of the black-hole perturbation mode) gravitational-electromagnetic black-hole quasinormal resonances with $M \Im \omega_{0}=0.01$. In particular, we display the dimensionless ratio $\Im \omega_{0} / \pi T_{\mathrm{BH}}$ for various values of the dimensionless black-hole charge parameter $Q / r_{+}$.

Interestingly, the numerical data presented in Table 1 provide compelling evidence that the relaxation rates of generic (that is, charged and rotating) Kerr-Newman black holes in the regime $Q / r_{+} \leq 0.9$ (this is the regime of black-hole electric charges studied numerically in [36]) are governed by the simple universal relation

$\Im \omega(n=0) \rightarrow \pi T_{\mathrm{BH}} \quad$ as $\quad T_{\mathrm{BH}} \rightarrow 0$. 


\section{Summary}

Our scientific knowledge about the resonance spectra of generic (that is, charged and rotating) Kerr-Newman black holes is not as good as our knowledge about the corresponding quasinormal spectra of rotating neutral Kerr black holes. This unsatisfactory state of affairs stems from the fact that all attempts to decouple the gravitational and electromagnetic perturbations of the Kerr-Newman black-hole spacetime have so far failed [8].

Thus, in order to study the coupled gravitationalelectromagnetic quasinormal spectra of generic KerrNewman black holes, one is forced to solve numerically a set of two coupled partial differential equations [8]. In this paper we have analyzed these numerically computed [36] black-hole resonances. In particular, we have provided compelling evidence that the characteristic relaxation times, $\tau \equiv 1 / \Im \omega_{0}$, of perturbed Kerr-Newman black-hole spacetimes in the regime $Q / r_{+} \leq 0.9$ (this is the regime of blackhole electric charges studied numerically in [36]) are characterized by the compact universal relation

$\tau \times T_{\mathrm{BH}} \rightarrow \pi^{-1}$ as $T_{\mathrm{BH}} \rightarrow 0$,

where $T_{\mathrm{BH}}$ is the Bekenstein-Hawking temperature of the Kerr-Newman black hole [see Eq. (3)]. The relation (5) suggests that neutral Kerr black holes and charged KerrNewman black holes share the same universal relaxation properties in the near-extremal $T_{\mathrm{BH}} \rightarrow 0$ regime.

We believe that it would be physically interesting (and mathematically challenging) to find an analytical explanation (it is worth emphasizing that Ref. [15] has already provided an analytical explanation for the simple asymptotic behavior (5) in the specific case of neutral near-extremal Kerr black holes) for the numerically suggested universal behavior (5) which characterizes the near-extremal Kerr-Newman blackhole spacetimes.

Acknowledgments This research is supported by the Carmel Science Foundation. I would like to thank Yael Oren, Arbel M. Ongo, Ayelet B. Lata, and Alona B. Tea for helpful discussions.

Open Access This article is distributed under the terms of the Creative Commons Attribution 4.0 International License (http://creativecomm ons.org/licenses/by/4.0/), which permits unrestricted use, distribution, and reproduction in any medium, provided you give appropriate credit to the original author(s) and the source, provide a link to the Creative Commons license, and indicate if changes were made.

Funded by SCOAP . $^{3}$

\section{References}

1. W. Israel, Phys. Rev. 164, 1776 (1967)

2. W. Israel, Commun. Math. Phys. 8, 245 (1968)

3. B. Carter, Phys. Rev. Lett. 26, 331 (1971)

4. S.W. Hawking, Commun. Math. Phys. 25, 152 (1972)

5. D.C. Robinson, Phys. Rev. D 10, 458 (1974)

6. D.C. Robinson, Phys. Rev. Lett. 34, 905 (1975)

7. J. Isper, Phys. Rev. Lett. 27, 529 (1971)

8. S. Chandrasekhar, The Mathematical Theory of Black Holes (Oxford University Press, New York, 1983)

9. R.P. Kerr, Phys. Rev. Lett. 11, 237 (1963)

10. E.T. Newman, R. Couch, K. Chinnapared, A. Exton, A. Prakash et al., J. Math. Phys. 6, 918 (1965)

11. H.P. Nollert, Class. Quantum Grav. 16, R159 (1999)

12. E. Berti, V. Cardoso, A.O. Starinets, Class. Quant. Grav. 26, 163001 (2009)

13. R.A. Konoplya, A. Zhidenko, Rev. Mod. Phys. 83, 793 (2011)

14. S.L. Detweiler, in Sources of Gravitational Radiation, ed. by L. Smarr (Cambridge University Press, Cambridge, 1979)

15. S. Hod, Phys. Rev. D 78, 084035 (2008). arXiv:0811.3806

16. S. Hod, Phys. Lett. B 715, 348 (2012). arXiv:1207.5282

17. S. Hod, Phys. Rev. D 75, 064013 (2007). arXiv:gr-qc/0611004

18. S. Hod, Class. Quant. Grav. 24, 4235 (2007). arXiv:0705.2306

19. A. Gruzinov, arXiv:0705.1725 [gr-qc]

20. S. Hod, Phys. Rev. D 78, 084035 (2008). arXiv:0811.3806

21. A. Pesci, Class. Quantum Grav. 24, 6219 (2007)

22. S. Hod, Phys. Lett. B 666, 483 (2008). arXiv:0810.5419

23. S. Hod, Phys. Rev. D 80, 064004 (2009). arXiv:0909.0314

24. S. Hod, Phys. Lett. A 374, 2901 (2010). arXiv:1006.4439

25. S. Hod, Phys. Rev. D. 84, 044046 (2011). arXiv: 1109.4080

26. S. Hod, Phys. Lett. B 710, 349 (2012). arXiv:1205.5087

27. S. Hod, Phys. Lett. B 715, 348 (2012). arXiv:1207.5282

28. S. Hod, Phys. Rev. D 88, 084018 (2013). arXiv: 1311.3007

29. S. Hod, Phys. Lett. B 747, 339 (2015). arXiv:1507.01943

30. S.A. Teukolsky, W.H. Press, Astrophys. J. 193, 443 (1974)

31. Z. Mark, H. Yang, A. Zimmerman, Y. Chen, Phys. Rev. D 91, 044025 (2015)

32. P. Pani, E. Berti, L. Gualtieri, Phys. Rev. Lett. 110, 241103 (2013)

33. P. Pani, E. Berti, L. Gualtieri, Phys. Rev. D 88, 064048 (2013)

34. M. Zilhão, V. Cardoso, C. Herdeiro, L. Lehner, U. Sperhake, Phys. Rev. D 90, 124088 (2014)

35. S. Hod, Euro. Phys. J. C (Lett.) 75, 272 (2015). arXiv:1410.2252

36. O.J.C. Dias, M. Godazgar, J.E. Santos, Phys. Rev. Lett. 114, 151101 (2015) 